\title{
Cardiovascular determinants of resuscitation from sepsis and septic shock
}

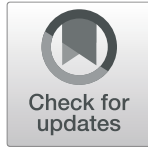

Fabio Guarracino ${ }^{1}$, Pietro Bertini ${ }^{1}$ and Michael R. Pinsky ${ }^{2 *}$ (D

\begin{abstract}
Background: We hypothesized that the cardiovascular responses to Surviving Sepsis Guidelines (SSG)-defined resuscitation are predictable based on the cardiovascular state.

Methods: Fifty-five septic patients treated by SSG were studied before and after volume expansion (VE), and if needed norepinephrine (NE) and dobutamine. We measured mean arterial pressure (MAP), cardiac index (CI), and right atrial pressure (Pra) and calculated pulse pressure and stroke volume variation (PPV and SW), dynamic arterial elastance (Eadyn), arterial elastance (Ea) and left ventricular (LV) end-systolic elastance (Ees), Ees/Ea (VAC), LV ejection efficiency (LVeff), mean systemic pressure analogue (Pmsa), venous return pressure gradient (Pvr), and cardiac performance (Eh), using standard formulae.

Results: All patients were hypotensive (MAP $56.8 \pm 3.1 \mathrm{mmHg}$ ) and tachycardic $\left(113.1 \pm 7.5\right.$ beat $\left.\mathrm{min}^{-1}\right)$, with increased lactate levels (lactate $=5.0 \pm 4.2 \mathrm{mmol} \mathrm{L}^{-1}$ ) with a worsened VAC. Cl was variable but $>2 \mathrm{~L} \mathrm{~min}^{-1} \mathrm{M}^{-2}$ in $74 \%$. Twentyeight-day mortality was $48 \%$ and associated with admission lactate, blood urea nitrogen (BUN), and creatinine levels but not cardiovascular state. In all patients, both MAP and Cl improved following VE, as well as cardiac contractility (Ees). Fluid administration improved Pra, Pmsa, and Pvr in all patients, whereas both HR and Ea decreased after VE, thus normalizing VAC. Cl increases were proportional to baseline PPV and SW. CI increases proportionally decreased PPV and SW. VE increased MAP > $65 \mathrm{mmHg}$ in 35/55 patients. MAP responders had higher PPV, SW, and Ea dyn than non-responders. NE was given to 20/55 patients in septic shock, but increased MAP > 65 mmHg in only 12. NE increased Ea, Eadyn, Pra, Pmsa, and VAC while decreasing HR, PPV, SW, and LVeff. MAP responders had higher pre-NE Ees and lower VAC. Dobutamine was given to 6/8 patients who remained hypotensive following NE. It increased Ees, MAP, Cl, and LVeff, while decreasing HR, Pra, and VAC. At all times and all steps of the protocol, $\mathrm{Cl}$ changes were proportional to Pvr changes independent of treatment.
\end{abstract}

Conclusions: The cardiovascular response to SSG-based resuscitation is highly heterogeneous but predictable from pretreatment measures of cardiovascular state.

Keywords: Heart-lung interactions, Blood volume, Ventriculo-arterial coupling, Effective circulating blood volume, Norepinephrine, Clinical trial

\section{Introduction}

Human sepsis is the result of a complex pathological process characterized by an infection-induced generalized intravascular inflammatory state causing marked dysregulation of cardiovascular adaptive responses [1,2]. Initial management, as defined by the Surviving Sepsis Guidelines (SSG), focuses on source control, early broad-spectrum antibiotics, and cardiovascular stabilization [3]. Systemic

\footnotetext{
*Correspondence: pinsky@pitt.edu

${ }^{2}$ Department of Critical Care Medicine, University of Pittsburgh, 1215.4 Kaufmann Medical Building, 3471 Fifth Avenue, Pittsburgh, PA 15213, USA Full list of author information is available at the end of the article
}

hypotension often persists following initial volume expansion (VE), independent of cardiac index (CI) characterizing generalized vasoplegia and ventricular dysfunction [2]. These complex and changing processes make the cardiovascular management of septic shock patients not only difficult but preclude simple formulaic approaches. Importantly, the fundamental pathological states defining human septic shock and its cardiovascular response to resuscitation therapies have not been described together. For example, levels of volume responsiveness, cardiac contractility, and ventriculo-arterial coupling (VAC) prior to resuscitation

(c) The Author(s). 2019 Open Access This article is distributed under the terms of the Creative Commons Attribution 4.0 International License (http://creativecommons.org/licenses/by/4.0/), which permits unrestricted use, distribution, and 
have been described, but not in the same subjects. Since there is much heterogeneity in individual patient cardiovascular reserve, we reasoned that there would be great value in linking disparate measures from different validated techniques into a common methodological approach and then applying a fixed generally recognized resuscitation protocol within this context. Intravascular fluid infusion to restore an adequate pressure gradient for venous return, vasop ressor-induced increased vasomotor tone to maintain organ perfusion pressure, and inotropes to improve cardiac contractility represent the three primary cardiovascular therapies used to restore cardiovascular stability in septic shock patients [3]. A more efficient and effective personalized resuscitation would greatly benefit from such an understanding of the patient's initial cardiovascular state, reserve, and response to resuscitation efforts.

We and others have documented the ability to estimate at the bedside of patients in septic shock mean systemic pressure (Pms) [4], left ventricular (LV) end-systolic elastance (Ees) [5], arterial elastance (Ea), and their associated derived cardiovascular parameters, allowing a deeper understanding of cardiovascular status and response to therapy. For example, based on Guytonian physiology, for cardiac output (CO) to increase, the pressure gradient for venous return (Pvr) (Pms minus right atrial pressure (Pra)) must increase [6], the resistance to venous return decrease [7], or both. Similarly, global cardiac efficiency (Eh) is quantified as the ratio of Pvr to Pms, with the more efficient heart increasing Pra less with fluid administration as $\mathrm{CO}$ increases [8]. Furthermore, the ratio of Ea to Ees, defining VAC [9], independently predicts outcomes in patients with cardiovascular disease [10] and is impaired in vasoplegic [11] and septic shock patients [12], suggesting that some of the septic myocardial depression reflects decoupling [13]. VAC is tightly linked to measures of LV ejection efficiency (LVeff), defined as the ratio of external work to total cardiac work during one cardiac cycle and is considered a major determinant of cardiac performance, being impaired if the $\mathrm{Ea} /$ Ees ratio varies too far in either direction from normal. Also, the dynamic measures, such as pulse pressure variation (PPV) or stroke volume variation (SVV), predict volume responsiveness [14], and the ratio of PPV to SVV, termed dynamic arterial elastance $\left(\mathrm{Ea}_{\mathrm{dyn}}\right)$, predicts the blood pressure response to changing cardiac output [15] or decreasing norepinephrine [16]. Thus, bedside clinicians have available to them an impressive array of potential cardiovascular diagnostic tests to define the cardiovascular state and reserve of their patients as well as accurately predict their response to specific therapies.

Although these powerful methods have been used separately to assess selected cardiovascular aspects of septic shock resuscitation, their combined analysis during protocolized resuscitation steps in septic patients is lacking. Thus, we analyzed the cardiovascular effects of stand ardized SSG resuscitation protocol [3] in septic patients. We hypothesized that the cardiovascular responses to resuscitation are predictable based on the cardiovascular state as quantified by these measured cardiovascular variables.

\section{Methods}

After ethical committee for human experimentation approval of both institutions, we enrolled 55 septic patients treated according to SSG [3]. Entry criteria included all patients sequentially presenting with presumed sepsis during the hours when the investigators (FG, $\mathrm{PB}$ ) were available. Sepsis was defined as a probable infectious etiology, hypotension (mean arterial pressure (MAP) $<65 \mathrm{mmHg}$ ), and lactic acidosis (lactate $>2 \mathrm{mmol} \mathrm{dL}^{-1}$ ). All patients who became stable signed informed consent, whereas those who never regained consciousness did not sign the consent but, as allowed by EEC rules, were still included in the analysis. We excluded patients $<18$ years of age and those patients with known significant mitral valve regurgitation and aortic valve or aortic pathologies because the arterial pressure signal was to be used to estimate CO.

Initial cardiovascular management included sequential $\mathrm{VE}$ with $30 \mathrm{~mL} / \mathrm{kg} 0.9 \mathrm{~N} \mathrm{NaCl}$ within $<3 \mathrm{~h}$ of enrollment, followed by norepinephrine infusion (NE), if needed to achieve an initial MAP $>65 \mathrm{mmHg}$ following VE, titrated between 0.01 and $1 \mu \mathrm{g} \mathrm{kg}^{-1} \mathrm{~min}^{-1}$, and then inotropic support with dobutamine was titrated between 5 and $15 \mu \mathrm{g} \mathrm{kg} \mathrm{min}^{-1}$, if evidence of hypotension and organ hypoperfusion persisted following NE, with all treatments given within $<5 \mathrm{~h}$ after enrollment. We limit norepinephrine to $1 \mu \mathrm{g} \mathrm{kg}{ }^{-1} \mathrm{~min}^{-1}$ to minimize the deleterious effects of high-dose vasopressors. All resuscitation decisions were made by the attending physicians. All patients had blood samples, appropriate body fluid, and other appropriate source cultures taken and received broad-spectrum antibiotics within $2 \mathrm{~h}$ of presentation and required mechanical ventilation. Prior to the initiation of the initial $30 \mathrm{~mL} \mathrm{~kg}^{-1}$ fluid bolus, we estimate that patients received between 250 and $500 \mathrm{~mL} 0.9 \mathrm{~N} \mathrm{NaCl}$ during the initial instrumentation.

Patients were instrumented with a central venous catheter, to infuse fluids and drugs and to measure Pra, a radial artery catheter to monitor MAP, to measure systolic arterial pressure, and by uncalibrated pulse contour analyses (MostCare ${ }^{\circ}$, Vygon, Italy) to continuously estimate CI, LV stroke volume, SVV, and PPV. Transthoracic echocardiography (TTE) was performed upon admission and then after each therapeutic intervention, if needed. Transesophageal echocardiography was used when TTE did not give sufficient images to assess cardiac function. Echocardiographic estimates of $\mathrm{CO}$ were used to assess the accuracy of the Mostcare ${ }^{\circ} \mathrm{CO}$ measures across treatments. Electrocardiograph (lead 2) RR intervals defined heart rate (HR) and peripheral pulse oximetry pulse oxygen saturation. Controlled mechanical 
ventilation was at a tidal volume of $6-8 \mathrm{mg} \mathrm{kg}^{-1}$ ideal body weight with a positive end-expiratory pressure/ $\mathrm{FiO}_{2}$ ratio as defined by ARDSnet guidelines [17]. Respiratory frequency was adjusted to end-tidal $\mathrm{CO}_{2}$ between 32 and $35 \mathrm{mmHg}$.

Derived hemodynamic parameters were calculated using standard formulae as illustrated in Fig. 1 and described in the Additional file 1: Methods. Briefly, LV Ees was calculated by the single beat method of Chen et al. and Ea as the ratio of 0.9.systolic arterial pressure and SV [5]. Ees zero pressure intercept defined Vo, and the potential energy (PE) per beat defined by the area of the Ees to end-systolic volume triangle [18]. LV stroke work (SW) was the area inside the LV pressure-volume loop estimated as the product of end-systolic pressure (ESP) and SV. LV pressure-volume area (PVA) was the sum of PE and SW, and the SW to PVA ratio defining LVeff [18]. Analogue Pms (Pmsa) was derived from paired end-expiratory $\mathrm{CO}$, Pra, and MAP measures by the method of Parkin and Leaning [8], and (Pmsa-Pra)/Pmsa defining cardiac performance (Eh). End-systolic and end-diastolic volumes (ESV and EDV) were measured by echocardiography. All echocardiographic measures were made by clinicians (FG, PB) expert in single beat Ees and Ea estimates.

\section{Statistical analysis}

To obtain a statistical power of 0.8 with alpha 0.5 and 95\% confidence intervals for the first enrollment were calculated a priori that we would need 42 patients [12]. Thus, we recruited over 50 patients to overcome any a priori un-estimated biases. All data was tested for normality using Shapiro-Wilk analysis, and paired sample $t$ test and Wilcoxon ranked sign test were carried out to assess differences induced by interventions. Independent sample $t$ test for normally distributed data and MannWhitney $U$ test for nonparametric measurements were used to test differences between variables grouped by MAP restored ( $>65 \mathrm{mmHg}$ ), MAP increased, and $\mathrm{CI}$ increased at $\geq 15 \%$. Pearson's correlation analysis was used to find correlation between normally distributed data, Spearman's correlation was used for nonparametric data, and chi-square test was used to find correlation between categorical data. Receiver operating characteristic analysis was used to test sensitivity and specificity of hemodynamic variables in predicting changes in categorical endpoints. Statistical studies were two-tailed, and $p$ value of less than 0.05 was considered significant. Analyses were performed using SPSS (v23 for Mac, IBM USA). Unless otherwise stated, data are presented as mean $\pm \mathrm{SD}$.

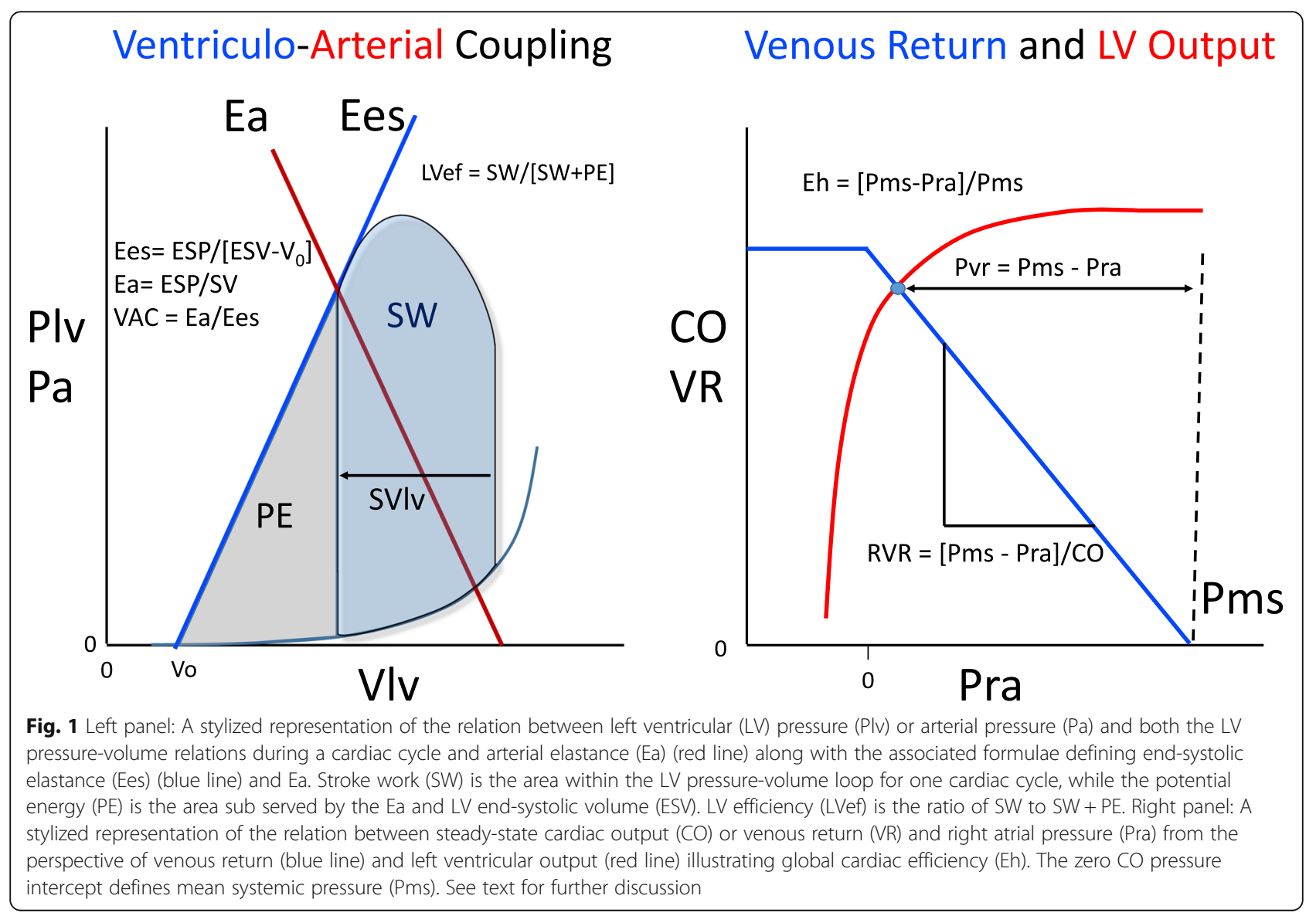




\section{Results}

Baseline pre-resuscitation status

Patient features on admission are described in Table 1. All were hypotensive (MAP $56.8 \pm 3.1 \mathrm{mmHg}$ ) and tachycardic $\left(113.1 \pm 7.5\right.$ beats $\left.\mathrm{min}^{-1}\right)$, with elevated lactate levels (lactate $5.0 \pm 4.2$ ) and had an increase in Ea relative to Ees consistent with ventriculo-arterial uncoupling. CI was variable, but in $74 \%$, CI was $>2 \mathrm{~L} \mathrm{~min}^{-1} \mathrm{M}^{-2}$. The mean admission SOFA score was 16.1, and the 30-day mortality rate was $47 \%$. Thirty-day mortality was associated with higher admission lactate, BUN, and creatinine levels, but not with any other measured or derived hemodynamic variables. Specifically, 35 patients resolved their hypotension with VE alone but retained a $44 \%$ 30-day mortality rate, whereas those in septic shock had a 52\% 30-day mortality rate of which was not significantly different. Additional file 2: Table S1 lists individual patient sepsis etiologies, SOFA and SAP II scores, and maximal doses of norepinephrine and dobutamine given.

Table 2 summarizes the cardiovascular parameters prior to and after the three sequential interventions, and Fig. 2a and $\mathrm{b}$ diagrams the associated mean ventriculo-arterial coupling and venous return-LV output changes while Additional file 3: Figure S1-S9 shows individual variables over each step per patient. In all subjects with all treatments, changes in $\mathrm{CO}$ co-varied in proportion to changes in Pvr (Fig. 3).

\section{Effect of VE}

The effects of VE on hemodynamic parameters are reported in Table 3. In all patients, both MAP and CI improved following VE, as well as cardiac contractility (Ees). Regarding Guytonian physiological parameters, fluid administration improved Pra, Pmsa, and Pvr in all patients. Moreover, both HR and Ea decreased, causing VAC to improve toward normal values, thus improving both LVeff and Eh. However, these hemodynamic changes were not uniformly observed across patients. Table 3 shows predefined subgroup analyses for $\mathrm{CI}$ and MAP responders and non-responders to each step of the protocol. Additional file 3: Figure S10 and S11 displays the $\mathrm{CI}$ responders (increase in $\mathrm{CI}>15 \%$ ) versus non-responders to a VE of $30 \mathrm{~mL} / \mathrm{kg}$ of crystalloids, NE and dobutamine, respectively; Fig. 4 displays the Ees and Ea

Table 1 Demographics of group on admission and relation to survival at 30 days

\begin{tabular}{|c|c|c|c|c|}
\hline Group variable & Total (mean \pm SD) & Alive at 30 days (mean \pm SD) & Dead at 30 days (mean \pm SD) & $p$ \\
\hline N & 55 & $29(53 \%)$ & $26(47 \%)$ & \\
\hline Age (years) & $69 \pm 11$ & $69 \pm 12$ & $71 \pm 11$ & ns \\
\hline Gender & M 34, F 21 & M 9, F21 & M 10, F 10 & ns \\
\hline MAP (mmHg) & $57 \pm 3$ & $57 \pm 3$ & $58 \pm 2$ & ns \\
\hline $\mathrm{Cl}\left(\mathrm{L} \min ^{-1} \mathrm{M}^{2}\right)$ & $2.09 \pm 0.11$ & $2.10 \pm 0.10$ & $2.04 \pm 0.11$ & ns \\
\hline $\mathrm{HR}\left(\mathrm{min}^{-1}\right)$ & $113 \pm 8$ & $112 \pm 8$ & $114 \pm 6$ & ns \\
\hline $\mathrm{Ea}\left(\mathrm{mmHg} \mathrm{mL^{-1 } )}\right.$ & $2.11 \pm 0.41$ & $2.10 \pm 0.36$ & $2.15 \pm 0.48$ & ns \\
\hline Ees $\left(m m H g ~ m L^{-1}\right)$ & $1.42 \pm 0.35$ & $1.36 \pm 0.37$ & $1.54 \pm 0.33$ & ns \\
\hline Ea/Ees & $1.56 \pm 0.41$ & $1.67 \pm 0.43$ & $1.44 \pm 0.39$ & ns \\
\hline Pra (mmHg) & $7.6 \pm 1.4$ & $7.5 \pm 1.5$ & $7.6 \pm 1.3$ & ns \\
\hline Pmsa (mmHg) & $13.0 \pm 1.4$ & $12.9 \pm 1.6$ & $12.9 \pm 1.3$ & ns \\
\hline Pvr (mmHg) & $5.3 \pm 0.5$ & $5.3 \pm 0.5$ & $5.3 \pm 0.5$ & ns \\
\hline Eh (\%) & $0.41 \pm 0.05$ & $0.42 \pm 0.05$ & $0.42 \pm 0.05$ & ns \\
\hline LV SW (mmHg mL) & $2581 \pm 900$ & $2842 \pm 740$ & $2414 \pm 1018$ & ns \\
\hline LV efficiency (\%) & $0.65 \pm 0.12$ & $0.65 \pm 0.12$ & $0.63 \pm 0.13$ & ns \\
\hline Lactate $\left(\mathrm{mmol} \mathrm{L}{ }^{-1}\right)$ & $5.1 \pm 4.3$ & $2.7 \pm 1.5$ & $7.1 \pm 4.8$ & $<0.005$ \\
\hline Hematocrit (\%) & $30.0 \pm 4.3$ & $30.0 \pm 4.3$ & $29.9 \pm 4.5$ & ns \\
\hline Hemoglobin $\left(\mathrm{g} \mathrm{dL}^{-1}\right)$ & $9.8 \pm 1.4$ & $9.8 \pm 1.4$ & $9.8 \pm 1.4$ & ns \\
\hline $\mathrm{BUN}\left(\mathrm{mg} \mathrm{dL}^{-1}\right)$ & $38.8 \pm 29.3$ & $27.3 \pm 15.2$ & $49.7 \pm 35.3$ & $<0.005$ \\
\hline Creatinine $\left(\mathrm{mg} \mathrm{dL}^{-1}\right)$ & $1.8 \pm 1.2$ & $1.3 \pm 0.9$ & $2.2 \pm 1.3$ & 0.010 \\
\hline Chronic hypertension & $26(47 \%)$ & $16(55 \%)$ & $10(38 \%)$ & ns \\
\hline SOFA score & $16 \pm 7$ & $11 \pm 5$ & $21 \pm 6$ & $<0.005$ \\
\hline SAPS II score & $63 \pm 15$ & $52 \pm 12$ & $74 \pm 8$ & $<0.005$ \\
\hline
\end{tabular}

Abbreviations: $M$ male, $F$ female, MAP mean arterial pressure, $C l$ cardiac index, $H R$ heart rate, Ea arterial elastance, Ees end-systolic elastance, Pra right atrial pressure, Pmsa mean systemic pressure analogue, Pvr pressure gradient for venous return, Eh global cardiac efficiency, $L V$ left ventricular, SW stroke work, $B U N$ blood urea nitrogen 
Table 2 Cardiovascular effects of resuscitation stages

\begin{tabular}{|c|c|c|c|c|c|c|}
\hline & Baseline & Volume expansion & Pre-norepinephrine & Norepinephrine & Pre-dobutamine & Dobutamine \\
\hline Fluid & - & $30 \mathrm{~mL} \mathrm{~kg}^{-1}$ & & - & & - \\
\hline NE & - & - & & $0.01-1 \mu \mathrm{g} \mathrm{kg}^{-1} \mathrm{~min}^{-1}$ & & $0.01-1 \mu \mathrm{g} \mathrm{kg}^{-1} \mathrm{~min}^{-1}$ \\
\hline Dobutamine & - & - & & - & & $5-15 \mu \mathrm{g} \mathrm{kg}^{-1} \mathrm{~min}^{-1}$ \\
\hline Patients & 55 & 55 & 20 & 20 & 8 & 8 \\
\hline Time to next step & 0 & $<3 \mathrm{~h}$ & & $<5 \mathrm{~h}$ & & $<5 h$ \\
\hline Variable & $x \pm S D$ & $x \pm S D$ & $x \pm S D$ & $x \pm S D$ & $x \pm \mathrm{SD}$ & $x \pm S D$ \\
\hline $\mathrm{SAP}(\mathrm{mmHg})$ & $81 \pm 20$ & $111 \pm 31^{*}$ & $95 \pm 26$ & $117 \pm 33^{*}$ & $100 \pm 28$ & $120 \pm 23$ \\
\hline $\mathrm{DAP}(\mathrm{mmHg})$ & $44 \pm 10$ & $44 \pm 15$ & $43 \pm 12$ & $35 \pm 14^{*}$ & $35 \pm 16$ & $40 \pm 11$ \\
\hline MAP (mmHg) & $57 \pm 3$ & $67 \pm 6^{*}$ & $60 \pm 2$ & $64 \pm 7^{*}$ & $57 \pm 2$ & $67 \pm 5^{*}$ \\
\hline PPV (\%) & $15.68 \pm 2.13$ & $11.47 \pm 1.24^{*}$ & $11.8 \pm 1.01$ & $10.85 \pm 0.99^{*}$ & $11.13 \pm 0.83$ & $8.76 \pm 3.89$ \\
\hline $\mathrm{Cl}\left(\mathrm{L} \min ^{-1} \mathrm{M}^{2}\right)$ & $2.09 \pm 0.11$ & $2.54 \pm 0.18^{*}$ & $2.47 \pm 0.17$ & $2.49 \pm 0.23$ & $2.39 \pm 0.26$ & $2.8 \pm 0.25$ \\
\hline $\mathrm{HR}\left(\min ^{-1}\right)$ & $113 \pm 8$ & $96 \pm 13^{*}$ & $109 \pm 8$ & $102 \pm 13^{*}$ & $116 \pm 7$ & $99 \pm 12^{*}$ \\
\hline $\mathrm{SV}(\mathrm{mL})$ & $35 \pm 5$ & $51 \pm 11^{*}$ & $43 \pm 7$ & $45 \pm 10$ & $38 \pm 5$ & $52 \pm 12^{*}$ \\
\hline SW (\%) & $18.45 \pm 3.04$ & $13.16 \pm 3.58^{*}$ & $15.9 \pm 4.32$ & $11 \pm 2.40^{*}$ & $11.13 \pm 3.36$ & $11.00 \pm 1.78$ \\
\hline $\mathrm{Ea}\left(\mathrm{mmHg} \mathrm{mL} \mathrm{L}^{-1}\right)$ & $2.11 \pm 0.41$ & $1.98 \pm 0.35^{*}$ & $1.97 \pm 0.37$ & $2.33 \pm 0.40^{*}$ & $2.38 \pm 0.41$ & $2.04 \pm 0.33$ \\
\hline Ees $\left(m m H g ~ m L^{-1}\right)$ & $1.42 \pm 0.35$ & $1.65 \pm 0.33^{*}$ & $1.50 \pm 0.3$ & $1.54 \pm 0.38$ & $1.18 \pm 0.20$ & $1.63 \pm 0.31^{*}$ \\
\hline $\mathrm{Ea} / \mathrm{Ees}$ & $1.56 \pm 0.41$ & $1.25 \pm 0.36^{*}$ & $1.38 \pm 0.44$ & $1.63 \pm 0.6^{*}$ & $2.10 \pm 0.62$ & $1.37 \pm 0.71^{*}$ \\
\hline $\operatorname{Pra}(\mathrm{mmHg})$ & $7.65 \pm 1.39$ & $8.53 \pm 1.53^{*}$ & $8.55 \pm 1.82$ & $9.32 \pm 1.76^{*}$ & $9.75 \pm 1.16$ & $6.6 \pm 1.27^{*}$ \\
\hline Pmsa (mmHg) & $12.98 \pm 1.44$ & $14.94 \pm 1.50^{*}$ & $14.5 \pm 1.79$ & $15.24 \pm 1.77^{*}$ & $15.41 \pm 1.54$ & $14.04 \pm 2.53$ \\
\hline Pvr (mmHg) & $5.32 \pm 0.49$ & $6.41 \pm 0.72^{*}$ & $5.95 \pm 0.59$ & $6.18 \pm 0.78$ & $5.66 \pm 0.75$ & $7.67 \pm 1.65^{*}$ \\
\hline Eh (\%) & $0.41 \pm 0.05$ & $0.43 \pm 0.06^{*}$ & $0.42 \pm 0.06$ & $0.41 \pm 0.07$ & $0.37 \pm 0.04$ & $0.54 \pm 0.05^{*}$ \\
\hline LV SW (mmHg mL) & $2581 \pm 900$ & $5299 \pm 2349^{*}$ & $3580 \pm 6120$ & $5023 \pm 2338^{*}$ & $6202 \pm 2381$ & $5845 \pm 2356^{*}$ \\
\hline LV efficiency (\%) & $0.65 \pm 0.12$ & $0.74 \pm 0.11^{*}$ & $0.71 \pm 0.17$ & $0.67 \pm 0.12$ & $0.65 \pm 0.17$ & $0.69 \pm 0.06$ \\
\hline
\end{tabular}

Abbreviations as in Table 1, plus, SAP systolic arterial pressure, DAP diastolic arterial pressure, $P P V$ pulse pressure variation, SV stroke volume, SVV stroke volume variation

${ }^{*} p<0.05$ with respect to the preceding resuscitation step

lines; and Fig. 5 displays the venous return differences between subgroups of responders and non-responders. CI increased by $>15 \%$ in 49 of 55 patients. The CI and MAP increases in response to VE and NE were proportional to the pre-treatment PPV and $\mathrm{Ea}_{\mathrm{dyn}}$ values (Additional file 3: Figure S12, S13, S14). As expected, VE-induced CI increases were associated with decreases in both PPV and SVV. Similarly, CI responders had higher baseline $\mathrm{Ea}_{\mathrm{dyn}}, \mathrm{Ea}$, and Ees than CI non-responders. Importantly, a VE of $30 \mathrm{~mL} / \mathrm{kg}$ of crystalloid also increased MAP $>65 \mathrm{mmHg}$ in 35 patients (MAP responders). CI responders $>15 \%$ (Fig. 4) increased SV, arterial pressure, and Ees, whereas non-responders only increased LV end-diastolic volume and SV. MAP responders had higher PPV, SVV, and $\mathrm{Ea}_{\mathrm{dyn}}$ than MAP non-responders. An $\mathrm{Ea}_{\mathrm{dyn}}<0.76$ predicted MAP non-responders (Fig. 6), whereas baseline $\mathrm{Ea}>1.9 \mathrm{~mL} \mathrm{mmHg}^{-1}$ was a weak though also significant predictor of MAP responders. Finally, patients with more normal baseline $\mathrm{Ea} /$ Ees had greater increases in $\mathrm{CI}$ and MAP. Additional subgroup analyses are given in Additional file 1: Results.

\section{Effect of NE}

$\mathrm{NE}$ was given to the 20 of 55 patients who remained hypotensive post VE. NE increased MAP in 12 patients by $10 \pm 2 \mathrm{mmHg}$ and decreased MAP slightly in 8 by $2 \pm 2$ $\mathrm{mmHg}$. NE was also associated with a decreased HR, PPV, and SVV, while Pra, Ea, $\mathrm{Ea}_{\mathrm{dyn}}$, and Pmsa increased. VAC worsened to decoupled values like those seen at baseline, whereas both Eh and LVeff were unchanged. Those 12 patients in whom MAP increased $>65 \mathrm{mmHg}$ (MAP responders) had higher pre-NE Ees and Ea values and lower HR than the MAP non-responders (Fig. 4c). An $\mathrm{Ea}_{\mathrm{dyn}}>0.83$ and $\mathrm{Ea}>1.75$ predicted a MAP increase $>$ 15\% (Fig. 6). Not surprisingly, the CI response to NE was also variable, increasing in 5 and minimally decreasing in 4 patients. Interestingly, NE CO responders increased Ees improving VAC whereas non-responders did not (Fig. 4c).

\section{Effect of dobutamine}

Dobutamine was given to 6 of the 8 patients whose MAP remained hypotensive following $\mathrm{NE}$ and VE. Dobutamine increased MAP, CI, and Ees causing VAC and Eh to improve. Both HR and Pra decreased, causing 

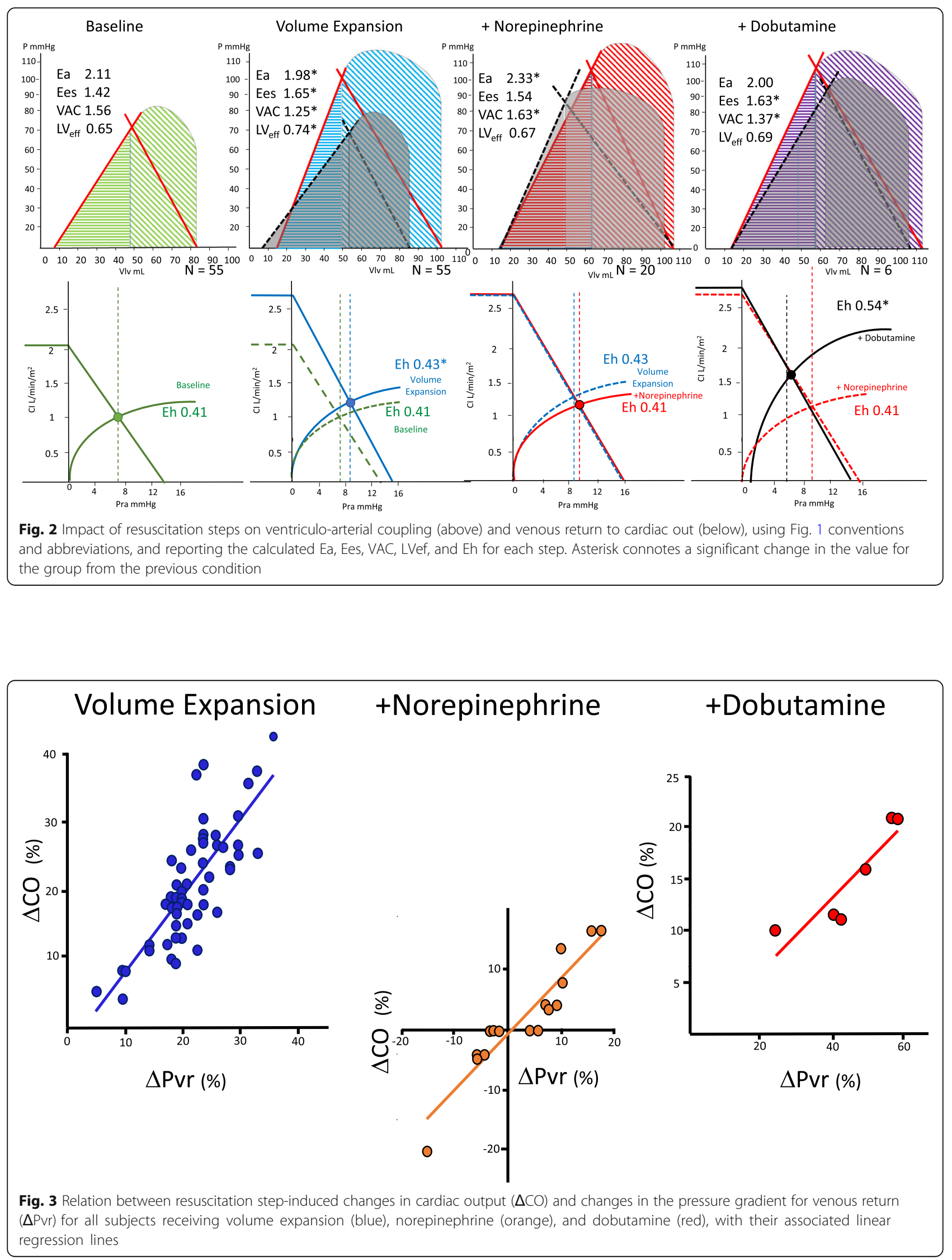
Table 3 Responder vs. non-responder subgroup analysis: cardiovascular effects of resuscitation

\begin{tabular}{|c|c|c|c|c|c|c|c|c|}
\hline \multirow{2}{*}{$\begin{array}{l}\text { MAP > } 65 \mathrm{mmHg} \\
\text { Variable }\end{array}$} & \multicolumn{2}{|l|}{ Baseline } & \multicolumn{2}{|c|}{ Volume expansion } & \multicolumn{2}{|l|}{ Norepinephrine } & \multicolumn{2}{|l|}{ Dobutamine } \\
\hline & Responders & Not responders & Responders & Not responders & Responders & Not responders & Responders & Not responders \\
\hline Patients & & & 35 & 20 & 12 & 11 & 6 & 2 \\
\hline SAP & $85 \pm 21$ & $74 \pm 18$ & $121 \pm 30^{*}$ & $95 \pm 27^{*}$ & $138 \pm 28^{*}$ & $100 \pm 28^{*}$ & $124 \pm 24$ & $102 \pm 25$ \\
\hline DAP & $43 \pm 10$ & $48 \pm 7$ & $44 \pm 16$ & $43 \pm 12^{*}$ & $35 \pm 13^{*}$ & $35 \pm 16^{*}$ & $43 \pm 11^{*}$ & $31 \pm 15$ \\
\hline MAP & $57 \pm 3$ & $57 \pm 3$ & $70 \pm 3^{*}$ & $60 \pm 2^{*}$ & $70 \pm 2^{*}$ & $57 \pm 2^{*}$ & $70 \pm 2^{*}$ & $60 \pm 6$ \\
\hline PPV & $16.8 \pm 1.1$ & $13.7 \pm 2.1$ & $11.3 \pm 1.3^{*}$ & $11.8 \pm 1.1^{*}$ & $10.7 \pm 1.1^{*}$ & $11.0 \pm 0.9^{*}$ & $9.6 \pm 3.5$ & $6.3 \pm 5.3$ \\
\hline $\mathrm{Cl}$ & $2.09 \pm 0.11$ & $2.10 \pm 0.11$ & $2.59 \pm 0.17^{*}$ & $2.47 \pm 0.17^{*}$ & $2.60 \pm 0.19^{*}$ & $2.39 \pm 0.26$ & $2.92 \pm 0.04^{*}$ & $2.20 \pm 0.31$ \\
\hline $\mathrm{HR}$ & $112 \pm 8$ & $115 \pm 6$ & $88 \pm 8^{*}$ & $108 \pm 8^{*}$ & $92 \pm 5^{*}$ & $114 \pm 8$ & $93 \pm 6^{*}$ & $115 \pm 7$ \\
\hline SV & $34.8 \pm 5.6$ & $34.2 \pm 3.8$ & $54.9 \pm 9.3^{*}$ & $42.9 \pm 6.5^{*}$ & $54.1 \pm 4.7^{*}$ & $37.5 \pm 5.4$ & $56.4 \pm 8.0^{*}$ & $32.8 \pm 6.2$ \\
\hline SW & $17.2 \pm 1.9$ & $20.7 \pm 3.4$ & $11.6 \pm 1.8^{*}$ & $15.9 \pm 4.3^{*}$ & $11.1 \pm 1.7^{*}$ & $11.0 \pm 3.2^{*}$ & $11.0 \pm 2.0$ & $11.0 \pm 1.4$ \\
\hline$E a_{d y n}$ & $1.00 \pm 0.13$ & $0.67 \pm 0.14$ & $0.99 \pm 0.15$ & $0.80 \pm 1.75^{*}$ & $0.98 \pm 0.14$ & $1.08 \pm 0.38^{*}$ & $0.88 \pm 0.44$ & $0.60 \pm 0.57$ \\
\hline Ees & $1.43 \pm 0.39$ & $1.40 \pm 0.28$ & $1.73 \pm 0.31^{*}$ & $1.50 \pm 0.30^{*}$ & $1.81 \pm 0.25^{*}$ & $1.19 \pm 0.19^{*}$ & $1.70 \pm 0.09^{*}$ & $1.40 \pm 0.71$ \\
\hline Ea & $2.20 \pm 0.38$ & $1.96 \pm 0.42$ & $1.98 \pm 0.34^{*}$ & $1.97 \pm 0.37$ & $2.24 \pm 0.37^{*}$ & $2.44 \pm 0.44^{*}$ & $1.93 \pm 0.12^{*}$ & $2.35 \pm 0.63$ \\
\hline Ea/Ees & $1.62 \pm 0.43$ & $1.44 \pm 0.36$ & $1.74 \pm 0.31^{*}$ & $1.38 \pm 0.44$ & $1.26 \pm 0.28$ & $2.12 \pm 0.59^{*}$ & $1.14 \pm 0.10^{*}$ & $2.05 \pm 1.49$ \\
\hline Pra & $7.9 \pm 1.2$ & $7.3 \pm 1.7$ & $8.5 \pm 1.4^{*}$ & $8.6 \pm 1.8^{*}$ & $9.0 \pm 2.1$ & $9.9 \pm 1.2^{*}$ & $6.8 \pm 1.5^{*}$ & $6.5 \pm 0.7$ \\
\hline Pmsa & $13.2 \pm 1.2$ & $12.6 \pm 1.8$ & $15.2 \pm 1.3^{*}$ & $14.5 \pm 1.8^{*}$ & $15.1 \pm 2.1^{*}$ & $15.4 \pm 1.5$ & $15.4 \pm 2.1$ & $11.7 \pm 1.7$ \\
\hline Pvr & $5.3 \pm 0.5$ & $5.3 \pm 0.4$ & $6.7 \pm 0.5^{*}$ & $6.0 \pm 0.6$ & $6.7 \pm 0.4^{*}$ & $5.7 \pm 0.8$ & $8.8 \pm 0.8^{*}$ & $5.7 \pm 0.8$ \\
\hline Eh & $0.41 \pm 0.05$ & $0.42 \pm 0.57$ & $0.44 \pm 0.05^{*}$ & $0.42 \pm 0.06$ & $0.45 \pm 0.06$ & $0.37 \pm 0.04^{*}$ & $0.57 \pm 0.04^{*}$ & $0.49 \pm 0.03$ \\
\hline SW & $2736 \pm 963$ & $2311 \pm 723$ & $6171 \pm 2284^{*}$ & $3722 \pm 1577^{*}$ & $6789 \pm 1923^{*}$ & $3480 \pm 1389$ & $6411 \pm 2131^{*}$ & $3019 \pm 978$ \\
\hline LV efficiency & $0.67 \pm 0.11$ & $0.61 \pm 0.12$ & $0.74 \pm 0.07^{*}$ & $0.73 \pm 0.15^{*}$ & $0.68 \pm 0.09$ & $0.66 \pm 0.15$ & $0.70 \pm 0.04$ & $0.60 \pm 0.11$ \\
\hline $\mathrm{Cl}>15 \%$ & \multicolumn{2}{|l|}{ Baseline } & \multicolumn{2}{|c|}{ Volume expansion } & \multicolumn{2}{|c|}{ Norepinephrine } & \multicolumn{2}{|l|}{ Dobutamine } \\
\hline Variable & Responders & Not Responders & Responders & Not Responders & Responders & Not Responders & Responders & Not Responders \\
\hline Patients & & & 49 & 6 & 7 & 14 & 5 & 3 \\
\hline SAP & $83 \pm 20$ & $66 \pm 22$ & $115 \pm 29^{*}$ & $81 \pm 34$ & $103 \pm 25$ & $118 \pm 34^{*}$ & $118 \pm 32$ & $122 \pm 18$ \\
\hline DAP & $44 \pm 10$ & $51 \pm 8$ & $43 \pm 15$ & $51 \pm 12$ & $46 \pm 1^{*}$ & $35 \pm 14^{*}$ & $46 \pm 14$ & $35 \pm 5$ \\
\hline MAP & $57 \pm 3$ & $56 \pm 2$ & $67 \pm 6^{*}$ & $61 \pm 4^{*}$ & $72 \pm 2$ & $62 \pm 7$ & $70 \pm 2^{*}$ & $64 \pm 8$ \\
\hline PPV & $16.0 \pm 1.8$ & $12.7 \pm 2.3$ & $11.4 \pm 1.3^{*}$ & $11.8 \pm 1.2$ & $11.0 \pm 1.4$ & $11.1 \pm 0.7^{*}$ & $11.3 \pm 0.6$ & $10.3 \pm 0.6$ \\
\hline $\mathrm{Cl}$ & $2.09 \pm 0.12$ & $2.07 \pm 0.52$ & $2.58 \pm 0.16^{*}$ & $2.28 \pm 0.12^{*}$ & $2.70 \pm 0.17^{*}$ & $2.45 \pm 0.23$ & $2.86 \pm 0.05^{*}$ & $2.70 \pm 0.44^{*}$ \\
\hline $\mathrm{HR}$ & $112 \pm 7$ & $119 \pm 5$ & $94 \pm 12^{*}$ & $108 \pm 11^{*}$ & $108 \pm 13$ & $105 \pm 14$ & $91 \pm 8$ & $102 \pm 16$ \\
\hline SV & $34.5 \pm 5.2$ & $34.9 \pm 2.9$ & $51.4 \pm 10.0^{*}$ & $43.2 \pm 8.9^{*}$ & $47.4 \pm 9.4$ & $44.6 \pm 9.9$ & $54.2 \pm 10.3^{*}$ & $50.7 \pm 15.7$ \\
\hline SW & $18.3 \pm 3.1$ & $19.7 \pm 2.0$ & $13.0 \pm 3.5^{*}$ & $14.3 \pm 4.2^{*}$ & $8.0 \pm 2.8$ & $11.3 \pm 2.8^{*}$ & $11.7 \pm 2.9$ & $10.3 \pm 0.6$ \\
\hline $\mathrm{Ea}_{\mathrm{dyn}}$ & $0.91 \pm 0.20$ & $0.65 \pm 1.56$ & $0.92 \pm 0.20$ & $0.89 \pm 0.27$ & $1.50 \pm 0.70$ & $1.04 \pm 0.31^{*}$ & $1.01 \pm 0.24$ & $1.00 \pm 0.10$ \\
\hline Ees & $1.44 \pm 0.37$ & $1.25 \pm 0.15$ & $1.67 \pm 0.34^{*}$ & $1.48 \pm 0.20^{*}$ & $1.70 \pm 0.20$ & $1.41 \pm 0.35$ & $1.70 \pm 0.10^{*}$ & $1.43 \pm 0.48$ \\
\hline Ea & $2.16 \pm 0.37$ & $1.70 \pm 0.48$ & $2.02 \pm 0.33^{*}$ & $1.65 \pm 0.34$ & $1.83 \pm 0.21$ & $2.36 \pm 0.36^{*}$ & $1.93 \pm 0.15$ & $2.27 \pm 0.46$ \\
\hline Ea/Ees & $1.57 \pm 0.39$ & $1.44 \pm 0.61$ & $1.27 \pm 0.36^{*}$ & $1.15 \pm 0.38$ & $1.09 \pm 0.17$ & $1.80 \pm 0.62^{*}$ & $1.14 \pm 0.12$ & $1.82 \pm 1.12$ \\
\hline Pra & $7.7 \pm 1.4$ & $7.2 \pm 1.3$ & $8.3 \pm 1.4^{*}$ & $10.5 \pm 1.2^{*}$ & $8.0 \pm 1.7$ & $9.3 \pm 1.7^{*}$ & $5.8 \pm 0.8^{*}$ & $7.3 \pm 1.5 *$ \\
\hline Pmsa & $13.1 \pm 1.4$ & $12.4 \pm 1.4$ & $14.8 \pm 1.5^{*}$ & $16.1 \pm 1.3^{*}$ & $14.5 \pm 1.4^{*}$ & $15.4 \pm 1.8^{*}$ & $13.2 \pm 1.9$ & $15.4 \pm 3.3$ \\
\hline Pvr & $5.3 \pm 0.5$ & $5.2 \pm 0.3$ & $6.5 \pm 0.7^{*}$ & $5.6 \pm 0.4^{*}$ & $6.5 \pm 0.3^{*}$ & $6.1 \pm 0.8$ & $7.4 \pm 1.6$ & $8.1 \pm 2.0$ \\
\hline Eh & $0.41 \pm 1.47$ & $0.42 \pm 0.04$ & $0.44 \pm 0.50^{*}$ & $0.35 \pm 0.03^{*}$ & $0.45 \pm 0.07^{*}$ & $0.40 \pm 0.06^{*}$ & $0.56 \pm 0.06^{*}$ & $0.52 \pm 0.04^{*}$ \\
\hline SW & $2639 \pm 902$ & $2115 \pm 808$ & $5535 \pm 2267$ & $3373 \pm 2294$ & $4507 \pm 1900$ & $4965 \pm 2415^{*}$ & $5932 \pm 2804$ & $5759 \pm 2448$ \\
\hline LV efficiency & $0.65 \pm 0.12$ & $0.62 \pm 0.08$ & $0.73 \pm 0.10^{*}$ & $0.80 \pm 0.14^{*}$ & $0.77 \pm 0.09$ & $0.66 \pm 0.12$ & $0.71 \pm 0.05$ & $0.66 \pm 0.06$ \\
\hline
\end{tabular}

Variable units and abbreviations as in Tables 1 and 2

*Statistically significant difference $(p<0.05)$ between mean variables values before and after each treatment split by positive response either in MAP $>65$ mmHg (first part of the table) or in $\mathrm{Cl}$ increase $>15 \%$ (second part of the table) 


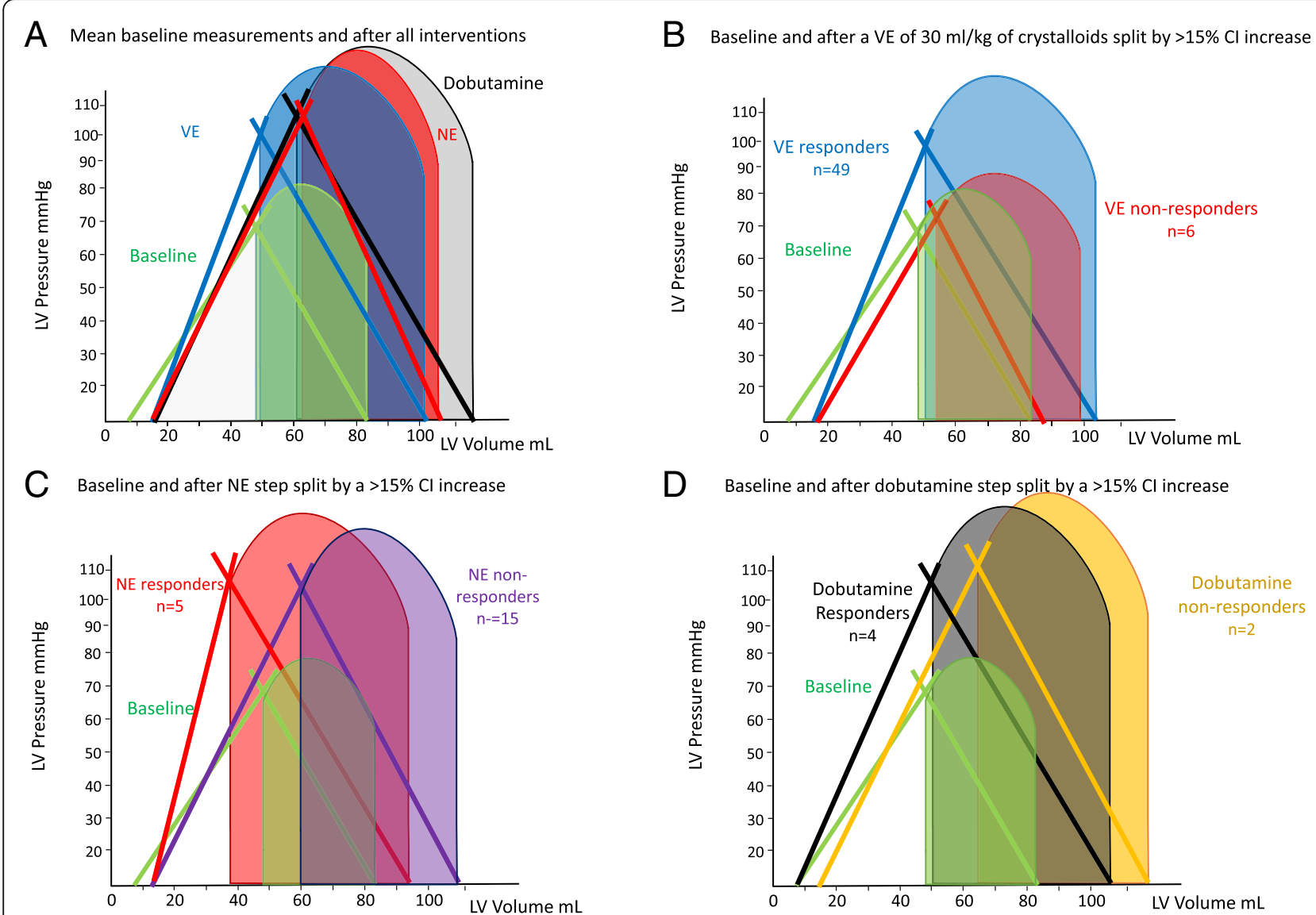

Fig. 4 Group difference ventriculo-arterial coupling between a mean values for all patients at baseline, and following $V E$, under $N E$, if needed, and then, if needed, under dobutamine. b Group differences in relations between those patients who increased their Cl >15\% to a VE of $30 \mathrm{~mL} /$ $\mathrm{kg}$ of crystalloids (responders) from non-responders. c Group differences in relations between those patients who increased their $\mathrm{Cl}>15 \%$ to NE (responders) from non-responders. $\mathbf{d}$ Group differences in relations between those patients who increased their $\mathrm{Cl}>15 \%$ to dobutamine (responders) from non-responders. The figure uses the same format, conventions, and abbreviations as Fig. 2 upper panel. LV left ventricle

Pvr to increase, whereas neither PPV, SVV, Pmsa, nor LVeff changed. Pre-dobutamine Ea was higher in those whose MAP increased (Ea $2.73 \pm 0.12$ vs. $2.16 \pm 0.37$ $\mathrm{mL} \mathrm{mmHg}{ }^{-1}, p=0.02$ ). Four of the 6 patients increased $\mathrm{CO}>15 \%$, and in those patients, Ees improved more than in the other $2 \mathrm{CO}$ non-responders (Fig. 4d).

\section{Discussion}

Our data demonstrate the heterogeneous cardiovascular profiles and responses to a SSG-defined resuscitation protocol in septic patients. Although our cohort received antibiotics within $2 \mathrm{~h}$ and $\mathrm{VE}$ within $3 \mathrm{~h}$ and completed the entire initial SSG protocol within $5 \mathrm{~h}$, we still observed a $47 \%$ 30-day mortality underscoring the lethality of septic shock. Interestingly, survival was associated with admission lactate, BUN, and creatinine levels but not with initial hemodynamic measures including responsiveness to VE or NE. These data also illustrate four principal cardiovascular findings.
First, that in most patients, VE restores MAP to $>65$ $\mathrm{mmHg}$ while simultaneously increasing $\mathrm{CO}$, Ees, and Pmsa, resulting in an improved VAC, LVeff, and Eh. Presumably, the increasing MAP improved coronary perfusion increasing Ees. Although Ees is a load-independent parameter of LV contractility, LV contractility is also improved by restoring coronary perfusion pressure, which occurred in these patients (Figs. 2 and 4b). Our results are also comparable to a large database analysis of septic shock patients $(n=3686)$ that reported only two thirds of patients being volume responders [19]. We enrolled patients prior to any fluid resuscitation other than an initial 250-500 mL fluid used during initial catheter insertion, which may not be the case with patients referred from the emergency department or ward where prior boluses of crystalloid fluids may have already been given. Thus, the percent volume responder may be less in a previously resuscitated cohort. Our data are also consistent with prior findings that PPV predicts $\mathrm{CO}$ responses to $\mathrm{VE}$ in septic patients [14] and that $\mathrm{Ea}_{\mathrm{dyn}}$ predicted the 


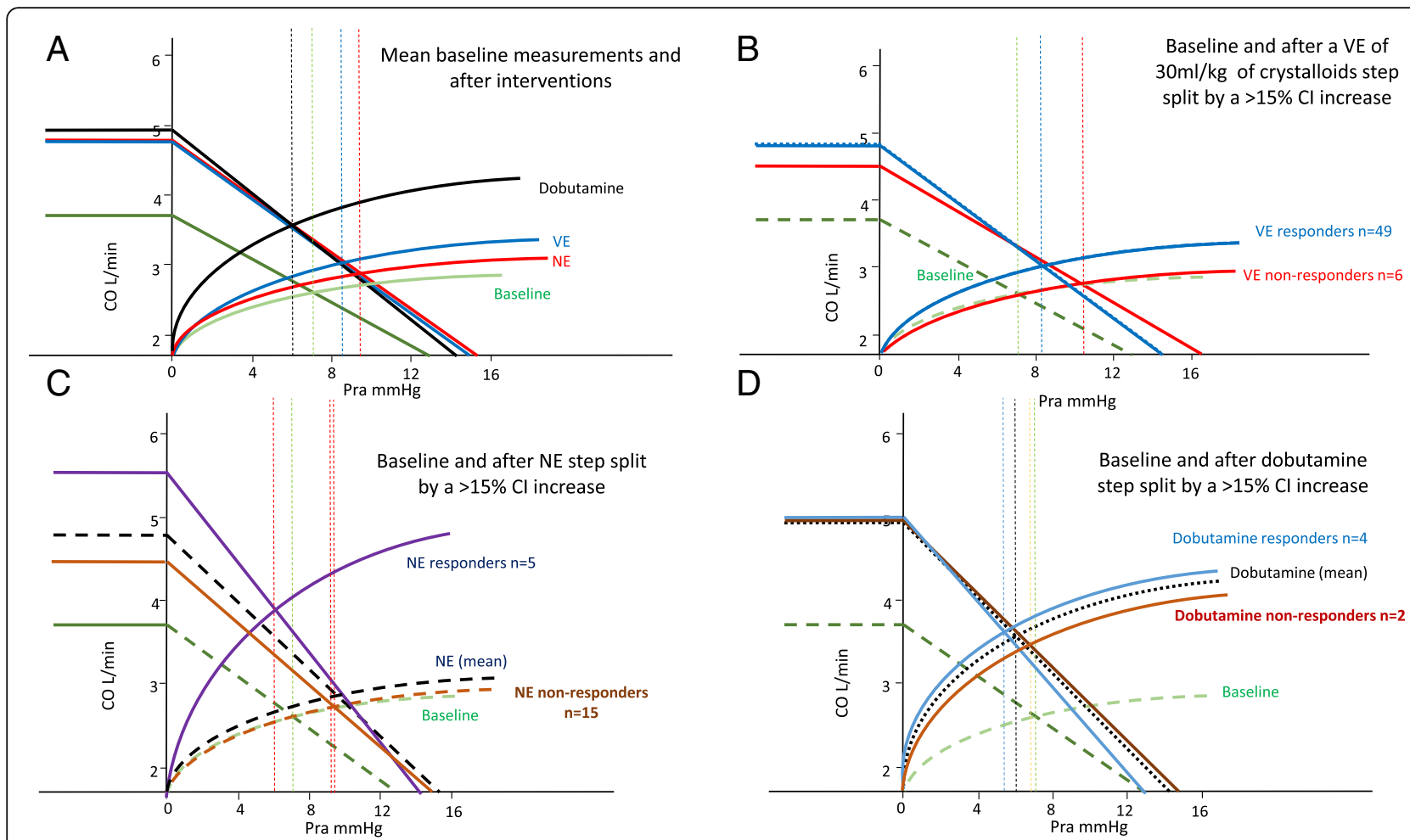

Fig. 5 Group differences in venous return to cardiac output relations between a mean values for all patients at baseline, and following VE, under $\mathrm{NE}$, if needed, and then, if needed under dobutamine. $\mathbf{b}$ Group differences in relations between those patients who increased their $\mathrm{Cl}>15 \%$ to a VE of $30 \mathrm{~mL} / \mathrm{kg}$ of crystalloids (responders) from non-responders. c Group differences in relations between those patients who increased their Cl $>15 \%$ to NE (responders) from non-responders. $\mathbf{d}$ Group differences in relations between those patients who increased their $\mathrm{Cl}>15 \%$ to dobutamine (responders) from non-responders. The figure uses the same format, conventions, and abbreviations as Fig. 2 bottom panel

associated change in MAP in response to changing $\mathrm{CO}$. [15] We found that individual patient MAP and CO responses to VE were variable, but accurately predicted by baseline PPV, SVV, and $\mathrm{Ea}_{\mathrm{dyn}}$, suggesting that fluid resuscitation based on these dynamic measures may result in more efficient fluid resuscitation by giving less fluid to non-responders. Several patients did not increase the CO in response to VE. Non-responders may become volume overloaded, with its associated increase morbidity [20]. These data also support the clinical relevance of using dynamic measures to guide fluid resuscitation in septic patients as recommended by the SSG [3]. Similarly, as CO increased, both PPV and SVV decreased, showing that PPV and SVV trending can be used clinically to monitor dynamic changes in cardiac output in response resuscitation maneuvers in septic patients (Fig. 6, Additional file 3: Figure S7, S8), as previously suggested [14].

Second, NE increased Ea and MAP in most patients but did not achieve a MAP $>65 \mathrm{mmHg}$ in a majority, consistent with prior studies of a variable response to norepinephrine in septic shock [21]. The pre-NE Ees, $\mathrm{Ea}$, and VAC predicted which patients would increase most their CO, suggesting that baseline Ees and VAC define the LV response to NE. Importantly, NE infusion also induced ventriculo-arterial uncoupling to levels seen prior to resuscitation (Additional file 3: Figure S6) and also decreased LVeff, which, if sustained, might impair LV performance (Figs. 2 and 4c). This data support the recent finding that sustained vasopressors use of $>6 \mathrm{~h}$ to maintain a MAP $>75 \mathrm{mmHg}$ in septic shock is associated with increased mortality [22]. However, it is unclear what level of VAC, its duration, or level of exogenous catecholamines are necessary to create this detrimental effect. Recently, animal studies demonstrate that NE can impair LV ejection by increasing the magnitude of arterial pressure reflected waves during ejection, which also becomes manifest as VAC uncoupling without increasing coronary perfusion pressure [23]. As was also seen in patients with postoperative vasoplegia [11], we observed that only patients with higher Ees and normalized VAC increased CO during NE (Fig. 4), presumably because they can tolerate the increased afterload.

Third, when dobutamine was added, VAC normalized while CO increased in all subjects. MAP simultaneously increased by a small but clinically significant amount (Fig. 4a, d). These data underscore the impact that exogenous inotropic support may improve contractility in septic patients who may be affected by septic cardiomyopathy [13]. These data also demonstrate the combined effect of dobutamine 

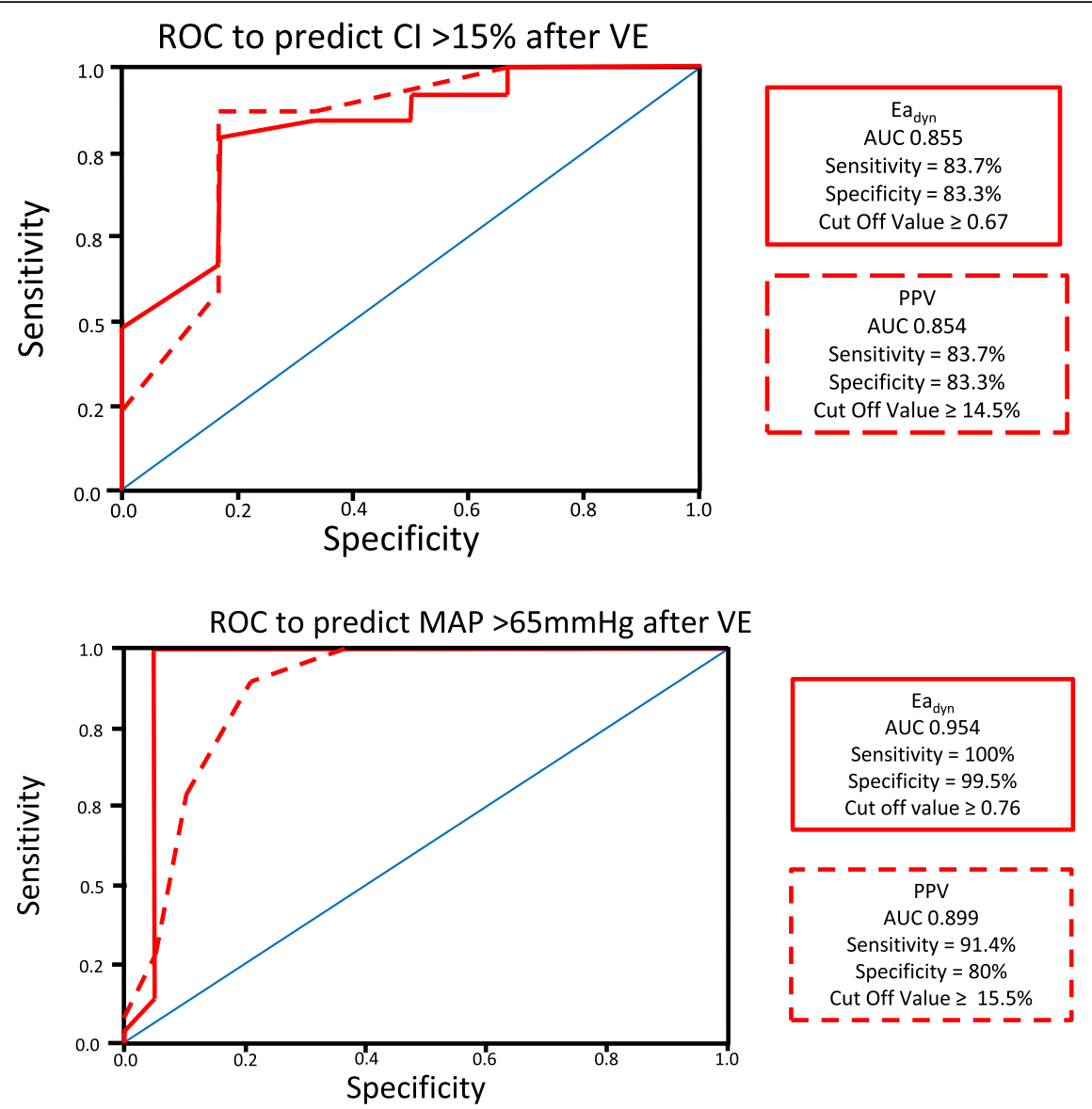

ROC to predict MAP $>15 \%$ after NE
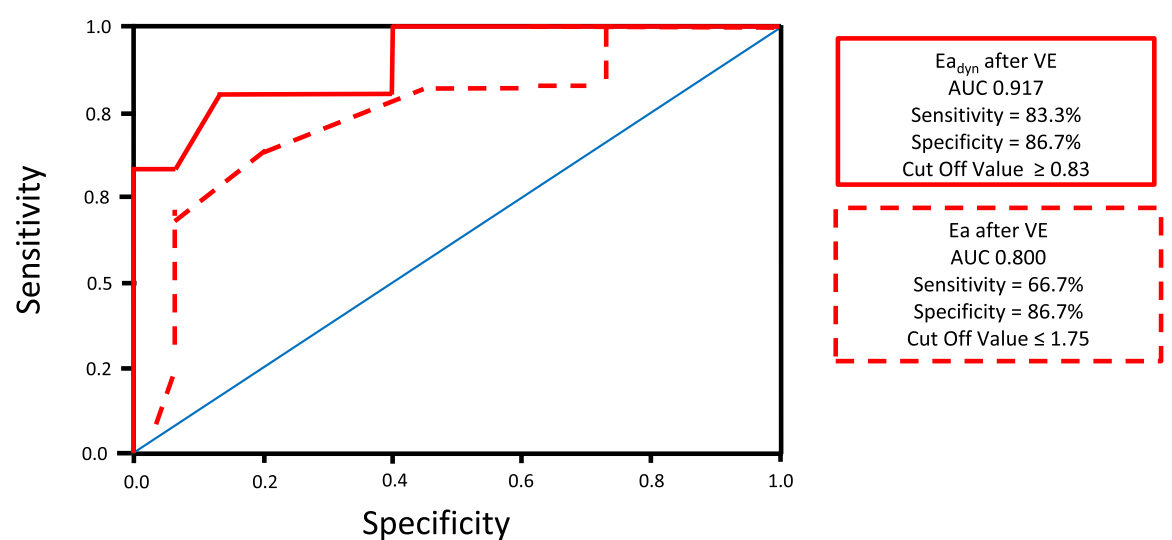

Fig. 6 Receiver operator characteristic curse for each value for the most informative predictive variable and its threshold value to predict VEinduced changes in cardiac index $(\triangle \mathrm{Cl})$ (upper panel) and MAP $(\triangle \mathrm{MAP}$ ) relative to baseline PPV and Eadyn (middle panel) for all patients and relation between $\triangle M A P$ in response to NE and PPV and $\mathrm{Ea}_{\mathrm{dyn}}$ for all subjects given NE (lower panel)

on both the arterial (Ea) and venous (Pmsa) circulations. Our data demonstrate that combined LV contractility and arterial tone processes, linked to venous return and LV performance [18], interact to define the arterial pressure and CO response to VE, NE, and dobutamine. Similar pooled cardiovascular parameter analyses to ours in future studies may increase our understanding of how other vasoactive and inotropic therapies, like vasopressin, angiotensin II or methylene blue infusions, and the use of low-dose corticosteroids, impact cardiovascular performance [21]. 
Fourth, at all times (VE, NE, dobutamine), changes in $\mathrm{CO}$ were proportional to changes in Pvr, independent of Pmsa changes (Fig. 3). These data are consistent with previous studies in postoperative surgical patients [24], stable septic patients being weaned from NE [25], and animals given dobutamine [7] and also validate that the Guytonian determinants of cardiovascular homeostasis [26] remain operative in septic patients during initial resuscitation. Specifically, VE and NE increased Pmsa and the subsequent change in $\mathrm{CO}$ was proportional to the change in Pvr. If NE decreased CO, it was because Pra increased more than Pmsa, as quantified by a decreasing Eh, whereas dobutamine, though decreasing both Pmsa and Pra, decreased Pra more, such that Pvr increased. Thus, modulating effective circulating blood volume with fluids and vasopressors is only one way to increase CO. Dobutamine increased $\mathrm{CO}$ by decreasing Pra. Importantly, septic patients still hypotensive during NE increased their MAP to dobutamine while restoring normal VAC, underscoring VAC as a fundamental determinant of MAP and $\mathrm{CO}$ responses in septic shock, as shown by others $[11,12,18]$.

\section{Limitations}

The study has several limitations. First, we did not personalize resuscitation therapy but used a defined SSG resuscitation protocol. Still, our findings agree with previous studies on VE responders [27] and support the use of dynamic parameters to predict $\mathrm{CO}$ and MAP responsiveness to VE $[14,15,25]$. Second, we only measured these cardiovascular variables during the initial 5-h resuscitation interval. Although we hypothesize that these parameters would continue to be predictive of cardiovascular response, other processes might decrease the predictive value of these measures. Still, during the initial rescue phase of septic shock [28], these parameters appear robust. Third, Ea, Ees, and Pmsa were calculated using formulae rather than measuring them directly using more invasive means. Thus, to the extent that the assumptions made to create these measures are inaccurate in severe sepsis, our measurement accuracy will degrade. For example, Ea is highly dependent on heart rate. Still, in our subjects, though tachycardic were unchanged during resuscitation. We doubt that other calculation inaccuracies exist because all these measures have been validated against more definitive measures in animal models of septic shock [29], and in humans during both septic shock [25] and post-cardiopulmonary bypass vasoplegia [30]. End-systolic pressure was estimated as 0.9.systolic arterial pressure measured at the radial artery. Systolic arterial pressure may be altered in sepsis as the measuring cite moves distal from the aorta. Still, in animal models, the subsequent change in systolic arterial pressure with resuscitation was similar across all measuring sites, meaning that the directional changes in calculated Ees and Ea would remain accurate independent of monitoring loci. Although we calculated Pmsa using formulae based on both $\mathrm{CO}$ and Pra [8], the computational components for Pra were an order of magnitude less than the observed changes in Pvr. Furthermore, we validated our Pmsa measures on endotoxic dogs using direct measures of Pms by transient stop flow during step-wise volume infusion and withdrawal, demonstrating an excellent correlation [29]. Furthermore, our Ea and Ees values compare to those of other studies $[11,12,18]$. Finally, we estimated $\mathrm{CO}$ and SV using an uncalibrated arterial pressure waveform analysis algorithm (Mostcare $\left.{ }^{\oplus}\right)$. To the extent that its assumptions become inaccurate during NE and dobutamine infusion, then the accuracy of these data will degrade. However, others have shown that the Mostcare $^{\oplus}$ device follows $\mathrm{CO}$ in septic shock patients given NE $[31,32]$. Furthermore, as listed in the methods, we checked the accuracy of the Mostcare ${ }^{\varpi} \mathrm{CO}$ estimates with echocardiographic estimates at each step and found the two methods consistent.

\section{Clinical perspective}

From the clinical perspective, several points become clear. First, it is impossible to know a priori if a septic patient who presents with hypotension fits the Sepsis-3 criteria for septic shock [33] without first performing $\mathrm{VE}$, as fully two thirds of our patients reversed their hypotension with the initial fluid resuscitation alone. Second, it is not clear if separating patients into either sepsis or septic shock is clinically relevant because the mortality rate in our cohort and their levels of end-organ injury was unaffected by this separation. Third, individualized fluid resuscitation based on the use of dynamic markers of fluid responsiveness allow for both accurate prediction of subsequent absolute change in cardiac output and, if followed continually, the actual changes in cardiac output over time. Fourth, although norepinephrine restores or sustained arterial pressure in septic shock patients, it does so at the expense of the heart, by selectively increasing $\mathrm{Ea}$ and decoupling LV ejection power from afterload. Thus, these data argue for limiting use of NE to initially restoring MAP and organ perfusion and then tapering it off once stable.

\section{Conclusion}

Initial fluid resuscitation based on SSG restores MAP and $\mathrm{CI}$ in most patients, while simultaneously restoring VAC and LVeff. However, individual patient responses vary widely. Similarly, the responses to NE in those persistently hypotensive or to subsequent dobutamine were also variable, but predictable based on their pre-treatment physiologic state. Finally, NE induced ventriculo-arterial decoupling which potentially may cause myocardial damage, if persistent. The bedside determination of Ea, Ees, and 
VAC plus dynamic parameters of volume responsiveness (e.g., PPV, SVV) and arterial tone $\left(\mathrm{Ea}_{\mathrm{dyn}}\right)$ is useful to both predict the responses to therapy and understand the differences in hemodynamic response among septic shock patients during resuscitation.

\section{Additional files}

Additional file 1: Supplemental Methods and Results (DOCX $25 \mathrm{~kb}$ )

Additional file 2: Table S1. Individual patient characteristics. (DOCX $13 \mathrm{~kb}$ )

Additional file 3: Figure S1. Relation between individual values of cardiac output (CO) over steps in the protocol: baseline, volume expansion (VE), plus norepinephrine (+NE), and plus dobutamine. Figure S2. Relation between individual values of mean arterial pressure (MAP) over steps in the protocol: baseline, volume expansion (VE), plus norepinephrine (+NE), and plus dobutamine. Figure S3. Relation between individual values of left ventricular end-systolic elastance (Ees) over steps in the protocol: baseline, volume expansion (VE), plus norepinephrine $(+\mathrm{NE})$, and plus dobutamine. Figure $\mathbf{S 4}$. Relation between individual values of arterial elastance (Ea) over steps in the protocol: baseline, volume expansion (VE), plus norepinephrine (+NE), and plus dobutamine. Figure S5. Relation between individual values of mean systemic pressure analogue (Pmsa) over steps in the protocol: baseline, volume expansion (VE), plus norepinephrine (+NE), and plus dobutamine. Figure S6. Relation between individual values of ventriculo-arterial coupling (VAC) from baseline to volume expansion (VE) to plus norepinephrine (+NE) with mean \pm SD for each step shown in blue. Values above 1.35 reflect uncoupling and values below 1.35 reflect normal VAC. Figure S7. Baseline to Volume Expansion relation between change in $\mathrm{CO}$ (DCO) and either pre-volume expansion pulse pressure variation (PPV) or dynamic arterial elastance $\left(E a_{d y n}\right)$. These data relate to the receiver operating characteristic results in Fig. 4. Figure S8. Baseline to volume expansion relation between change in mean arterial pressure (DMAP) and either pre-volume expansion pulse pressure variation (PPV) or dynamic arterial elastance $\left(E_{d y n}\right)$. These data relate to the receiver operating characteristic results in Fig. 4. Figure S9. Volume Expansion to Norepinephrine relation between change in mean arterial pressure (DMAP) and either pre-norepinephrine arterial elastance (Ea) or dynamic arterial elastance $\left(E_{d y n}\right)$. These data relate to the receiver operating characteristic results in Fig. 4. (PPTX 395 kb)

\section{Acknowledgements}

None.

\section{Funding}

None.

\section{Availability of data and materials}

All data collected in this study is summarized within the text and supplemental material sections freely available to the readers.

\section{Authors' contributions}

$\mathrm{FG}, \mathrm{PB}$, and MRP helped develop the study design, analyzed the data, and prepared and approved the manuscript for publication. FG and PB recruited the patients and made the measurement on the patients. All authors read and approved the final manuscript.

\section{Ethics approval and consent to participate}

The study was approved by both institutions' review boards for human experimentation. All subjects who regained consciousness signed informed consent while those who did not did not sign but by Italian law were permitted to be included in this study.

\section{Consent for publication}

The authors agree to waive copyright and allow this manuscript to be published in Critical Care.

\section{Competing interests}

The authors declare that they have no competing interests.

\section{Publisher's Note}

Springer Nature remains neutral with regard to jurisdictional claims in published maps and institutional affiliations.

\section{Author details}

${ }^{1}$ Department of Anesthesia and Critical Care Medicine, Azienda Ospedaliero Universitaria Pisana, Pisa, Italy. ${ }^{2}$ Department of Critical Care Medicine, University of Pittsburgh, 1215.4 Kaufmann Medical Building, 3471 Fifth Avenue, Pittsburgh, PA 15213, USA.

Received: 23 January 2019 Accepted: 29 March 2019

Published online: 15 April 2019

\section{References}

1. Angus DC, van der Poll T. Severe sepsis and septic shock. N Engl J Med. 2013;369:840-51.

2. Singer M, Deutschman CS, Seymour CW, Shankar-Hari M, Annane D, Bauer M, Bellomo R, Bernard GR, Chiche J-D, Coopersmith CM, Hotchkiss RS, Levy MM, Marshall JC, Martin GS, Opal SM, Rubenfeld GD, van der Poll T, Vincent $J \mathrm{~L}$, Angus DC. The third international consensus definitions for sepsis and septic shock (Sepsis-3). JAMA. 2006;315:801-10.

3. Rhodes A, Evans LE, Alhazzani W, Levy MM, Antonelli M, Ferrer R, Kumar A Sevransky JE, Sprung CL, Nunnally ME, Rochwerg B, Rubenfeld GD, Angus DC, Annane D, Beale RJ, Bellinghan GJ, Bernard GR, Chiche JD, Coopersmith C, De Backer DP, French CJ, Fujishima S, Gerlach H, Hidalgo JL, Hollenberg SM, Jones AE, Karnad DR, Kleinpell RM, Koh Y, Lisboa TC, Machado FR, Marini JJ, Marshall JC, Mazuski JE, Mclntyre LA, McLean AS, Mehta S, Moreno RP, Myburgh J, Navalesi P, Nishida O, Osborn TM, Perner A, Plunkett CM, Ranieri M, Schorr CA, Seckel MA, Seymour CW, Shieh L, Shukri KA, Simpson SQ, Singer M, Thompson BT, Townsend SR, Van der Poll T, Vincent IL, Wiersinga WJ, Zimmerman JL, Dellinger RP. Surviving sepsis campaign: international guidelines for management of sepsis and septic shock: 2016. Intensive Care Med. 2017:43:304-77.

4. Maas JJ, Geerts BF, de Wilde RBC, van den Berg PCM, Pinsky MR, Jansen JRC. Assessment of venous return curve and mean systemic filling pressure in post-operative cardiac surgery patients. Crit Care Med. 2009;37:912-8.

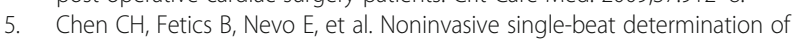
left ventricular end-systolic elastance in humans. J Am Coll Cardiol. 2001;38: 2028-34.

6. Cecconi M, Aya HD, Geisen M, Ebm C, Fletcher N, Grounds M, Rhodes A. Changes in the mean systemic filling pressure during a fluid challenge in postsurgical intensive care patients. Intensive Care Med. 2013;39:1299-305.

7. Geerts BF, Maas JJ, Lagrand WK, Aarts LP, Pinsky MR, Jansen JRC. Partitioning the resistances along the vascular tree: effects of dobutamine and hypovolemia in piglets with an intact circulation. J Clin Monit Comp. 2010;24:377-84.

8. Parkin WG, Leaning MS. Therapeutic control of the circulation. J Clin Monit Comput. 2008;22:391-400.

9. Sunagawa K, Maughan WL, Burkhoff D, Sagawa K. Left ventricular interaction with arterial load studied in isolated canine ventricle. Am J Phys. 1983;245:H773-80

10. Ky B, French B, May Khan A, Wang A, Chirinos JA, Fang JC, Sweitzer NK, Borlaug BA, Kass DA, Sutton MSJ, Cappola TP. Ventricular-arterial coupling remodeling, and prognosis in chronic heart failure. J Am Coll Cardiol. 2013; 62:1165-72.

11. Guinot $\mathrm{PG}$, Longrois $\mathrm{D}$, Kamel S, Lorne E, Dupont H. Ventriculo-arterial coupling analysis predicts the hemodynamic response to norepinephrine in hypotensive postoperative patients: a prospective observational study. Crit Care Med. 2018:46:e17-25.

12. Guarracino F, Ferro B, Morelli A, Bertini P, Baldassarri R, Pinsky MR. Arterioventricular decoupling in human septic shock. Crit Care. 2014;18:R80.

13. Rudiger A, Singer M. Mechanisms of sepsis-induced cardiac dysfunction. Crit Care Med. 2007;35:1599-608.

14. Michard F, Boussat S, Chemla D, Anguel N, Mercat A, Lecarpentier Y, Richard C, Pinsky MR, Teboul J-L. Relation between respiratory changes in arterial pulse pressure and fluid responsiveness in septic patients with acute circulatory failure. Am J Respir Crit Care Med. 2000;162:134-8. 
15. Monge Garcia Ml, Gil Cano A, Gracia Romero M. Dynamic arterial elastance to predict arterial pressure response to volume loading in preloaddependent patients. Crit Care. 2011;15:R15.

16. Guinot PG, Bernard E, Levard M, Dupont H, Lorne E. Dynamic arterial elastance predicts mean arterial pressure decrease associated with decreasing norepinephrine dosage in septic shock. Crit Care. 2015;19:14.

17. Network ARDS. Ventilation with lower tidal volumes as compared with traditional tidal volumes for acute lung injury and the acute respiratory distress syndrome. N Engl J Med. 2000;342:1301-8.

18. Burkdoff D. Pressure-volume loops in clinical research. J Am Coll Cardiol. 2013;62:1173-6.

19. Leisman DE, Doerfler ME, Schneider SM, Masick KD, D'Amore JD, D'Angelo JK. Predictors, prevalence, and outcomes of early crystalloid responsiveness among initially hypotensive patients with sepsis and septic shock. Crit Care Med. 2018:46:189-98.

20. Payen D, de Pont AC, Sakr Y, Spies C, Reinhart K, Vincent UL. A positive fluid balance is associated with a worse outcome in patients with acute renal failure. Crit Care. 2008;12:R74.

21. Levy B, Fritz C, Tahon E, Jacquot A, Auchet T, Kimmoun A. Vasoplegic treatment: the past, the present, and the future. Crit Care. 2018;22:52.

22. Lamontagne F, Day AG, Meade MO, Cook DJ, Guyatt GH, Hylands M, Radermacher P, Chretien JM, Beaudoin N, Hebert P, D'Argon F, Meziani F, Asfar P. Pooled analysis of higher versus lower blood pressure targets for vasopressor therapy in septic and vasodilatory shock. Intensive Care Med. 2018;44:12-21.

23. Monge-Garcia MI, Jian Z, Settles JJ, Hunley C, Cecconi M, Hatib F, Pinsky MR. Performance comparison of ventricular and arterial $\mathrm{dP} / \mathrm{dtmax}$ for assessing left ventricular systolic function during different experimental loading and contractile conditions. Crit Care. 2018;22:325.

24. Maas JJ, Pinsky MR, de Wilde RB, de Jonge E, Jansen JR. Cardiac output response to norepinephrine in postoperative cardiac surgery patients: interpretation with venous return and cardiac function curves. Crit Care Med. 2013:41:143-50.

25. Persichini R, Silva S, Teboul JL, Jozwiak M, Chemla D, Richard C, Monnet X. Effects of norepinephrine on mean systemic pressure and venous return in human septic shock. Crit Care Med. 2012;40:3146-53.

26. Guyton AC. Regulation of cardiac output. Anesthesiology. 1968;29:314-26.

27. Pruinelli L, Westra BL, Yadav P, Hoff A, Steinbach M, Kumar V, Delaney CW, Simon G. Delay within the 3-hour surviving sepsis campaign guideline on mortality for patients with severe sepsis and septic shock. Crit Care Med. 2018;46:500-5.

28. Starling MR. Left ventricular-arterial coupling relations in the normal heart. Am Heart J. 1993;125:1659-66.

29. Lee J, Ogedele O, Pike F, Pinsky MR. Effect of acute endotoxemia on analogue estimates of mean systemic pressure. J Crit Care. 2013;28:880.e9-15.

30. Guinot PG, Abou-Arab O, Guilbart M, Bar S, Zogheib E, Daher M, et al. Monitoring dynamic arterial elastance as a means of decreasing the duration of norepinephrine treatment in vasoplegic syndrome following cardiac surgery: a prospective, randomized trial. Intensive Care Med. 2017; 43:643-51.

31. Frachi F, Silvestri R, Cubattoli L, Taccone FS, Donadello K, Romano SM, et al. Comparison between an uncalibrated pulse contour method and thermodilution technique for cardiac output estimation in septic patients. Br J Anaesth. 2011;107:202-8.

32. Scolletta S, Franchi F, Romagnoli S, Carla R, Donati A, Fabbri LP, et al. Comparison between Doppler-echocardiography and uncalibrated pulse contour method for cardiac output measurement: a multicenter observational study. Crit Care Med. 2016;44:1370-9.

33. Singer M, Deutschman CS, Seymour CW, Shankar-Hari M, Annane D, Bauer $M$, et al. The third international consensus conference definitions for sepsis and septic shock (Sepsis-3). JAMA. 2016;315:801-10.

Ready to submit your research? Choose BMC and benefit from:

- fast, convenient online submission

- thorough peer review by experienced researchers in your field

- rapid publication on acceptance

- support for research data, including large and complex data types

- gold Open Access which fosters wider collaboration and increased citations

- maximum visibility for your research: over $100 \mathrm{M}$ website views per year

At BMC, research is always in progress.

Learn more biomedcentral.com/submissions 